\title{
ADHESION AND FRICTION MEASUREMENT METHOD FOR A MEMS PROBE ARRRAY
}

\author{
Wesley S. Smith, Peter G. Hartwell, Robert G. Walmsley \\ Hewlett-Packard Laboratories \\ Palo Alto, CA 94304
}

\begin{abstract}
This paper presents a unique method for measuring the adhesion and friction forces between a MEMS probe tip array and a sample surface. The method includes capability to measure the dependence of stiction and friction with varying normal force, humidity, surface materials, and rest time. A differential laser Doppler vibrometer (LDV) is used to measure the relative velocity between an unconstrained sample and the probe tips $(20 \mathrm{~nm}$ radius of curvature). By removing lateral constraints, only frictional forces act in the direction of relative motion. We present preliminary results comparing the adhesion forces to the normal force and examining time dependence of adhesion. No-load adhesive forces $(6-20 \mathrm{nN}$ per probe tip), coefficients of static friction (0.4-0.56), and trends in time dependence are compared to previous findings indicating that valid results are obtained with this method.
\end{abstract}

\section{INTRODUCTION}

Adhesion and friction forces are present any time two surfaces contact each other. Advancing technology in microdevices has led to several applications in which the movement of one surface over another is required. Applications involving probe arrays are being investigated for use in data storage [1], parallel imaging [2], and biological analysis [3]. Other applications where adhesion and friction play dominant roles include scanning probe microscopy [4], micro-motors and gears [5], and contacting stops of micro-mirrors [6]. Several studies have shown that the adhesive and friction forces depend on the normal force between the surfaces, the contact area, and the relative humidity [4-10]. Understanding the effects of these conditions on micro-scale adhesion and friction can lead to increased control of movement, knowledge of forces required for actuators, and investigation of failure mechanisms such as wear.

\section{MICRO AND NANO SCALE FRICTION}

Micro devices concentrate contact to a single asperity or controlled locations of asperities. Due to high surface area to volume ratios van der Waals forces, electrostatic forces, and capillary forces dominate over inertial and gravitational forces [8]. At this scale, interfacial friction can be described by

$$
F=S_{c} A+\mu N .
$$

The $\mu N$ term is the traditional Amontons' formulation for macroscale friction, and the $S_{c} A$ term represents the dependence on the contact area between the surfaces. Here $S_{c}$ is the critical shear stress between the two layers [9]. Other sources suggest adhesion forces are also strongly dependent on meniscus bridges that form in the presence of humidity at contacting surfaces [7-9].

Wear resulting from friction and adhesion is an issue for MEMS applications such as data storage [1,11]. Tip wear depends on the material properties of the contacting surfaces and the normal loads between them [11]

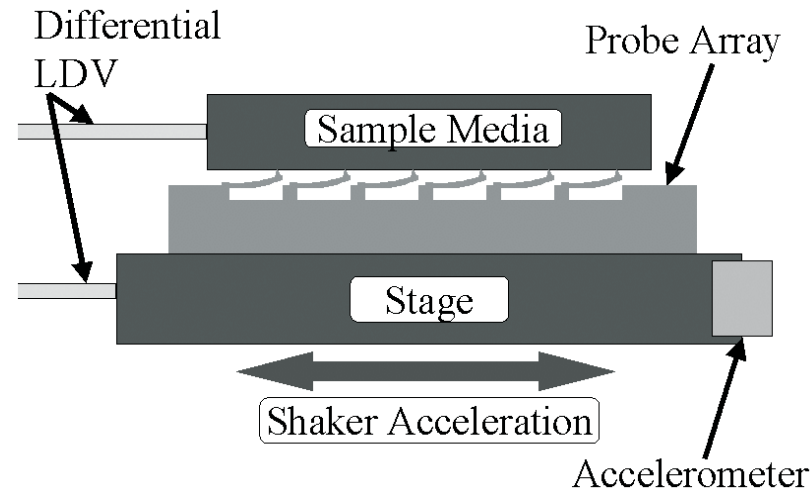

Figure 1. Schematic of measurement setup. The unconstrained sample media sits on top of an array of probe tips that are located at the end of cantilevers. Acceleration of the stage creates lateral friction forces that act on the sample media at the probe tips.

Methods used for measuring friction on the micro-scale generally involve a form of scanning probe microscopy including atomic force microscopy (AFM), friction force microscopy (FFM), scanning tunneling microscopy (STM) [4], or surface force apparatus (SFA)[12]. Scanning methods monitor the reactions of a cantilever as a probe tip is moved over a surface. SFA involves measuring adhesive forces by contacting a probe to a surface and measuring the force required to pull the probe from the surface. These methods have been used to characterize friction in several varying conditions including velocity, relative humidity, normal load, contact area, and behavior over asperities [8-10]. The results from these experimental methods depend on precise calibration of piezoelectric actuators used to provide the force for the scanning and accurate characterization of the cantilevers $[8]$.

We developed a unique method to characterize the scale dependent adhesion and friction effects between a MEMS probe tip array and a sample surface. This technique provides flexibility to vary the effective normal force, ambient humidity, contact media, and time intervals between actuated motions, allowing accurate characterization of these effects on friction. The experimental setup eliminates unknown reaction forces associated with scanning a cantilever over a fixed surface and the necessary calibrations required by other friction measurement methods. The system shown in Figure 1 isolates adhesion and friction forces by reducing the forces that act on the sample to the normal force at the probe tips, the sample's weight do to gravity, and the lateral friction forces.

\section{EXPERIMENTAL SETUP}

Adhesion and friction forces are measured between an array of probe tips pointing upward and a flat sample media that is free to move on top of the tips (Figure 1). The single crystal silicon (SCS) probe tips are located at the end of silicon cantilevers. A pre-stressed nitride layer causes the cantilevers to bend upwards 

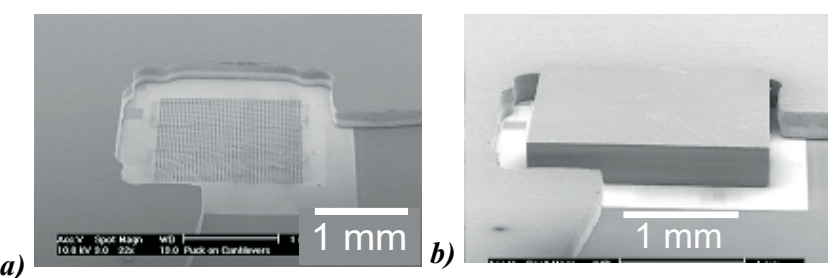

c)
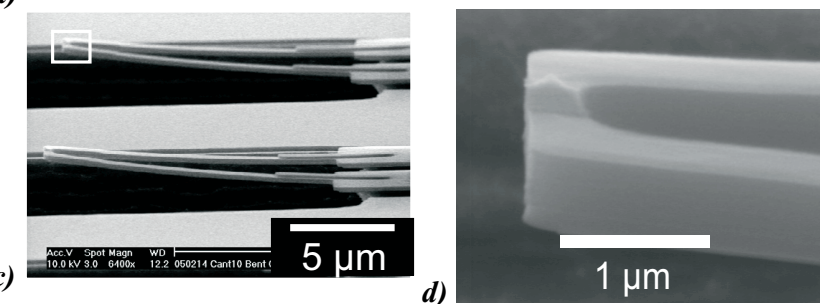

Figure 2. (a) SEM images of probe array with stainless steel fence, (b) the $2 \mathrm{~mm} \times 2 \mathrm{~mm} \times 380 \mu \mathrm{m}$ sample media on top of the

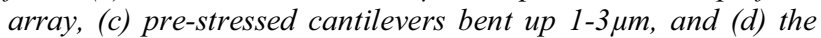
oxidation sharpened probe tips ( $\sim 20 \mathrm{~nm}$ radius of curvature).

providing a $1-3 \mu \mathrm{m}$ gap between the sample and the substrate (Figure 2a-c). Variations in the amount the cantilevers are bent up and differences in the tip heights are compensated for by the compliance of the individual cantilevers. Uniform contact between the sample surface and the probe tips is achieved as the cantilevers deflect due to the weight of the sample. At this scale, surface forces dominate over any small variations in normal force present at each probe tip. The complete array contains 1600 (40x40 array) probe tips that are oxidation sharpened to have a nominal radius of curvature of $20 \mathrm{~nm}$ (Figure 2d). The array spans an area of $1.6 \mathrm{~mm} \mathrm{x}$ $1.6 \mathrm{~mm}$. The sample media is a SCS chip $(2 \mathrm{~mm} \times 2 \mathrm{~mm} \times 380 \mu \mathrm{m})$ that is placed on top of the probe tips (Figure $2 b$ ).

The array of cantilevers is rigidly fixed to a horizontal shaker by gluing the array to a stainless steel fence (Figure 2a,b). This fence fixes the array to the shaker stage, constrains the sample to remain above the probe array, and allows an optical path to the sample's edge for vibrometer measurements. The cantilever orientation can be set to point in the direction of acceleration (axial) or perpendicular to the acceleration (transverse). The shaker is capable of producing horizontal accelerations $\left(>40 \mathrm{~m} / \mathrm{s}^{2}\right)$ more than sufficient to overcome the stiction forces. An accelerometer mounted to the stage shown in Figure 3 measures the motion of the shaker.

A differential laser Doppler vibrometer (LDV) focused on the shaker stage and on the edge of the silicon sample measures the relative motion of the sample and the probe array (Figure 1,3). The sample will remain fixed to the probe array until the lateral force of the shaker overcomes the stiction force. Dynamic friction governs the subsequent relative motion.

The experimental setup shown in Figure 3 includes a nozzle that is calibrated to vary the normal force between the probes and the sample and an environmental chamber to vary the ambient relative humidity. An equivalent normal force between the probe tips and the sample can be varied between 30 and $250 \mu \mathrm{N}$ using a pressure transducer to control the flow rate of nitrogen through the vertically oriented nozzle (Figure 3 ). The set-point pressure to equivalent mass conversion is calibrated by positioning the nozzle over a microbalance (Figure 4). An exponential equation is fit to the calibration data for finding intermediate equivalent masses. The effective normal force is the product of gravity and the sum of the sample mass and the equivalent mass. The dependence of stiction and friction on normal force is obtained by measuring the relative motion while varying the pressure applied to the top of the sample.

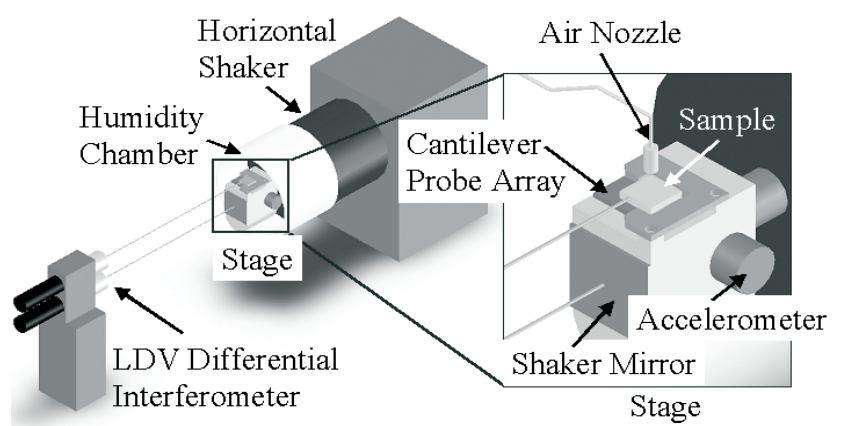

Figure 3. Overall schematic of friction test setup. LDV lasers are focused on a mirror attached to the shaker and on the edge of the sample media resting on top of the probe tips. A nozzle is located directly above the sample media to provide a calibrated additional normal force to the sample media $(30-250 \mu N)$. An environmental chamber surrounds the entire setup providing relative humidity control between 0.5 and $75 \%$ RH.

Previous studies have shown a strong dependence of friction characteristics on ambient humidity [4-10]. An environmental chamber encloses the shaker's stage to control the humidity at the contact points. Adjusting the flow rate of nitrogen over a water surface in a bubbler controls the relative humidity. The nozzle described above is also equipped with vacuum to lift the sample off the probe tips. This was done when humidity was changed to ensure uniform distribution of humidity between the probe tips and the sample. A hygrometer on the outlet line measures the relative humidity inside the chamber. Values of relative humidity between $0.5 \%$ and $75 \%$ were obtained using this method.

Additional flexibilities of this measurement method include the ability to quantify the time dependence of stiction and to vary the materials being examined. A custom actuation waveform was applied to the shaker to examine rest time effects (Figure 5b). By varying the time between impulse events $(50-500 \mathrm{~ms})$ within the waveform and the time between triggering the waveform $(30 \mathrm{~s}-$ $10 \mathrm{~min})$, the characteristics of the time dependence can be determined. Although not performed in these experiments, the sample media can be coated with various materials including polymers or thin films to measure the friction characteristics of these materials. In addition, thin films can be grown on the probe tips for friction characterization between two desired materials.

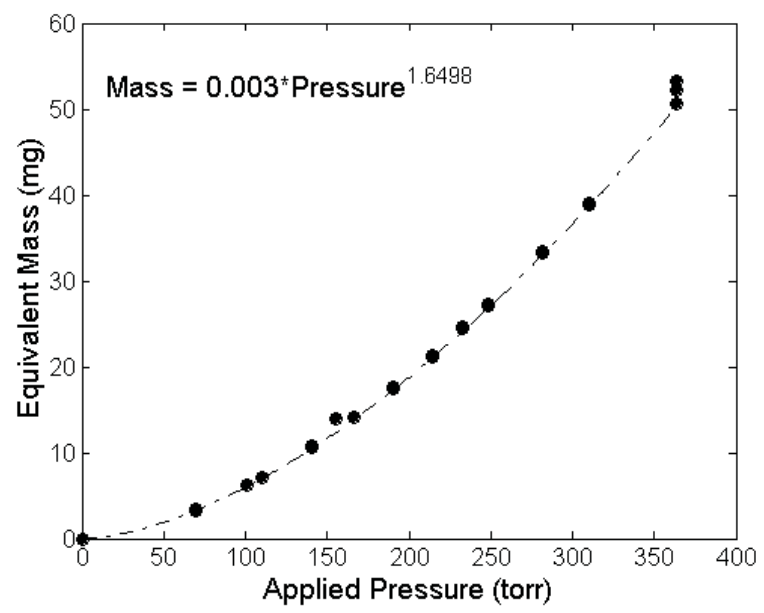

Figure 4. Equivalent mass calibration for the pressure controlled nozzle. An exponential fit was applied to determine intermediate equivalent mass values. 


\section{EXPERIMENTAL METHODS}

Verification that the sample is in uniform contact with the probe tips is done by analyzing the vertical deflection of the sample when hit with a burst of pressure. The differential LDV measures the vertical displacement of the sample and the pressure transducer gives data on the amount of pressure applied. Comparing the force-deflection curves gives the spring constant of the array. Consistent spring constants at each of the four corners of the sample indicate that uniform tip contact has been achieved.

Two forms of shaker actuation are used in the experiments. The first method involves a continuously oscillating acceleration that steadily increases in magnitude (Figure 5a). Relative motion of the sample to the probe array occurs when the acceleration exceeds the critical force needed to overcome stiction. After this release, the dynamic friction at the probe tips governs the motion of the sample. A major disadvantage of this method is that it involves a high travel distance between the sample and the tips that contribute to wearing of the tips and changes in the behavior of friction.

The second actuation method applies several bursts of acceleration sufficient in magnitude to overcome stiction. This method removes the hysteresis effects of stiction on time by accelerating the probe array twice in the same direction (pulses 2 and 3 in Figure 5b). It also reduces the travel distance between the two surfaces to minimize wear and allows adjustment of the time between pulses to examine the time dependence of stiction.

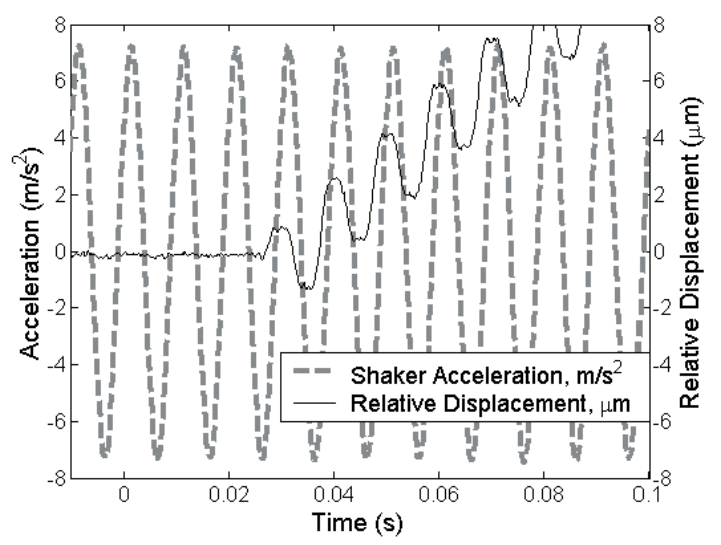

a)

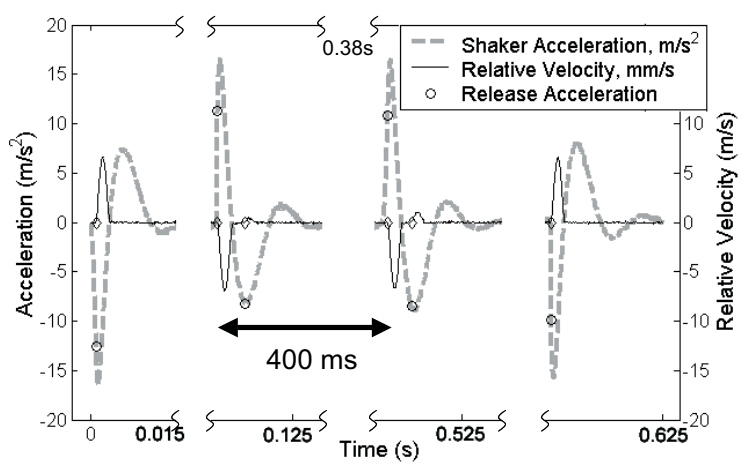

Figure 5. Continuous (a) and pulsed (b) actuation waveforms are plotted with the characteristic output from the differential $L D V$. The bias of movement in (a) is due to the axial orientation of the cantilevers (cantilevers point in same direction as acceleration). This bias is removed by rotating the cantilevers $90^{\circ}$. A rest time of $400 \mathrm{~ms}$ is shown in (b).

\section{RESULTS}

Preliminary results were obtained for various samples involving a single crystal silicon (SCS) sample in contact with SCS probe tips. Figures 6 and 7 show the normal load dependence of adhesion. The results in Figure 6 compare the two different actuation methods using two different probe arrays.

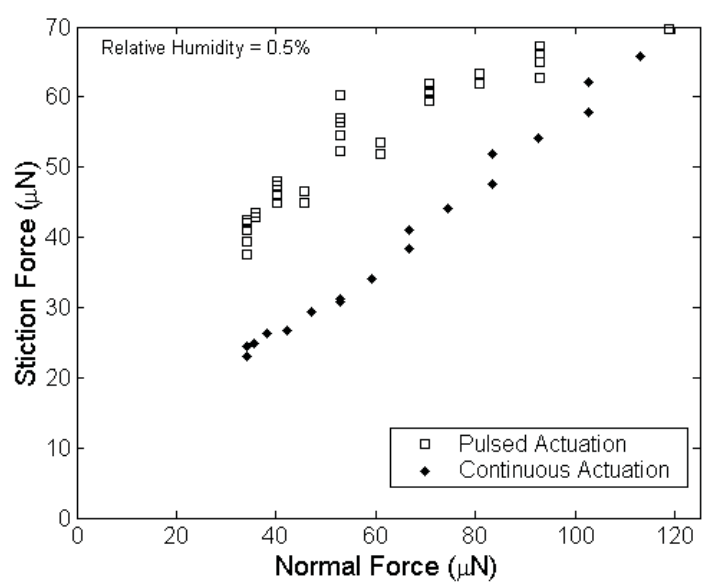

Figure 6. Dependence of stiction on normal force. Force values represent the force over the complete array of 1600 probe tips.

The slope of the lines in the plot above gives the coefficient of static friction. With a linear fit to the data, a static coefficient of 0.40 to 0.56 is found for the silicon surface sliding over the probe array. These values are consistent with the microscale friction coefficients of $0.45-0.50$ for $\mathrm{Si}(100)$ reported by Tambe and Bhushan [7]. As the normal load approaches zero, JKR theory predicts a non-zero stiction force [9]. This no-load adhesive force is purely dependent on the surface area of the two contacting surfaces. Extrapolating the data shown in Figure 6 to zero normal force gives no-load adhesive forces of $6-20 \mathrm{nN}$ per cantilever tip ( $20 \mathrm{~nm}$ radius of curvature). These values correspond well with the adhesive forces of $50 \mathrm{nN}$ reported for a $30-50 \mathrm{~nm}$ radius of curvature tip $[\mathbf{8 , 1 0}]$.

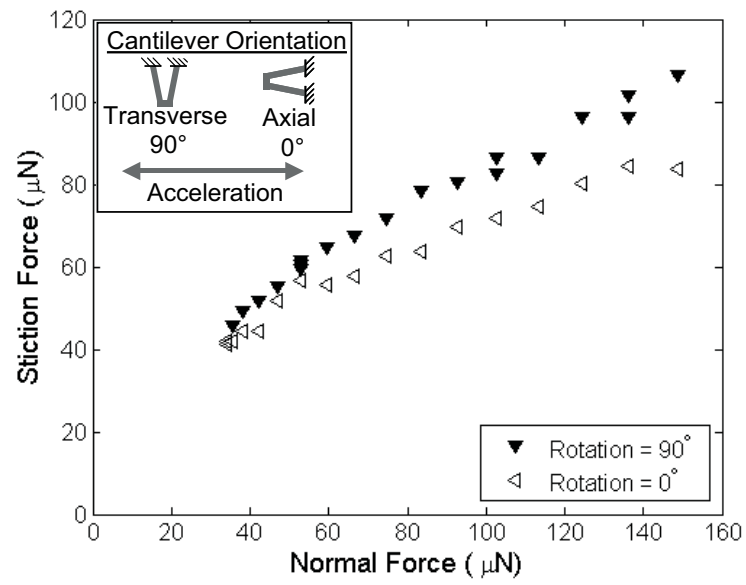

Figure 7. Effect of orientation of cantilevers on stiction force with pulsed actuation. Rotating the cantilevers $90^{\circ}$ produces higher friction forces at higher loads. The forces are distributed over the array of 1600 probe tips. 
Figure 7 shows the effect of the orientation of the cantilevers with respect to the direction of acceleration. When the cantilevers point in the same direction as the acceleration (axial), there is a slight bias between the stiction forces in one direction (Figure 5a). When the cantilevers are oriented perpendicular to the acceleration (transverse), this bias is removed, and the friction forces are shown to increase.

Results for the time dependence of stiction using the pulsed actuation method are shown in Figure 8. At small rest times between releases $(50-500 \mathrm{~ms})$, the values for friction show very little dependence on time. However, at longer rest times, there is a trend for the stiction value to increase slightly. This increase is uniform for both relatively dry conditions (RH $0.5 \%$ ) and ambient conditions $(\mathrm{RH}=40 \%)$. The total increase in static friction force per cantilever is on the order of $1.5 \mathrm{nN}$ over a rest time of $400 \mathrm{~s}$. This magnitude of increase is much smaller than published single tip results where there is approximately a $30 \mathrm{nN}$ change over the same period $[\mathbf{8}]$.

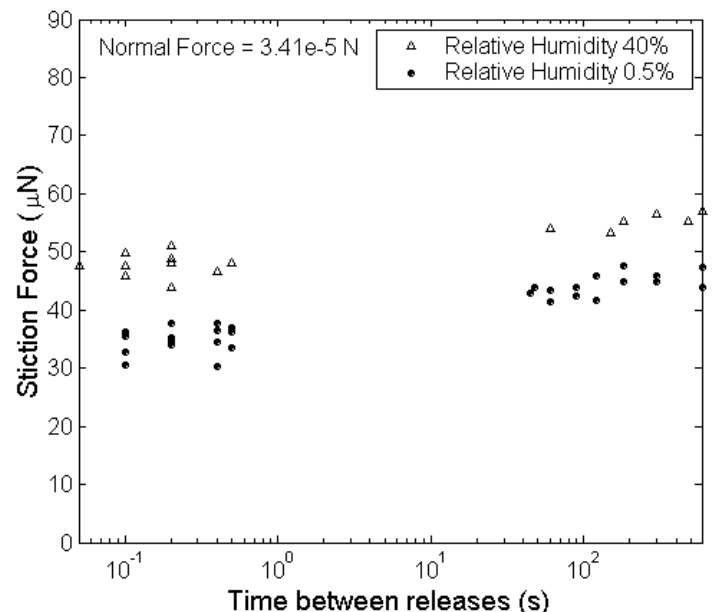

Figure 8. Time dependence of stiction for axially oriented cantilevers. The time dependence of stiction is shown to have a slightly increasing trend toward more stiction with greater time. Although the stiction is higher for $\mathrm{RH}=40 \%$, the trend is similar for dry conditions. Stiction forces are shown over the 1600 probe tip array.

\section{CONCLUSION}

The methods for measuring micro and nano-scale friction between a MEMS probe tip array and a sample surface presented in this paper produce similar results to those using scanning probe microscopy. By removing the constraints on the sample surface, lateral motion is governed solely by friction forces. This method also avoids calibration of piezo actuators needed for the force measurements of the scanning probe techniques. However, several complications to the method exist. Since friction is measured across an entire array, average values of friction forces are found. The strong dependence on surface contact area causes small fabrication variations to produce large differences in resulting forces.

The method described here is intended to serve as a platform for future studies of friction. It provides the flexibility to examine many of the main factors currently attributed to adhesion and friction. Further studies are underway to characterize the adhesion force dependence on relative humidity. The method will incorporate a vacuum chamber replacing the current environmental chamber to examine friction in dry conditions. For studies in the reliability of MEMS devices where friction and wear are issues, further investigations on the behavior of different material coatings and quantifying the wear that occurs are needed.

\section{ACKNOWLEDGEMENTS}

The authors would like to thank Hewlett-Packard Laboratories for supporting this work. Special thanks goes to Uija Yoon, now at Stanford University, for the fabrication of the probe arrays. We would also like to thank Matt Messana of Stanford University for contributing data filters for the analysis of the stiction data and Tom Kenny of Stanford University for his support in preparing this manuscript.

\section{REFERENCES}

[1] P. Vettiger,.G. Cross, M. Despont, U. Drechsler, U. Durig, B.Gotsmann, W. Haberle, M.A. Lantz, H.E. Rothuizen, R. Stutz, and G.K. Binnig, "The 'Millipede'-Nanotechnology Entering Data Storage". IEEE Trans. On Nanotech., 91, 1 (2002).

[2] S. C. Minne, G. Yaralioglu, S. R. Manalis, J. D. Adams, J. Zesch, A. Atalar, C. F. Quate, "Automated parallel high-speed atomic force microscopy," App. Phys. Lttrs. 72, 18 (1998).

[3] M. Poggi, L.A. Bottomley, and P.T. Lillehei. "Scanning Probe Microscopy" Anal. Chem. 74 (2002).

[4] B. Bhushan, H. Fuchs, S. Hosaka. "Micro/nanotribology studies using scanning probe microscopy," Applied Scanning Probe Mehods. Springer-Verlag, Berlin Heidelberg (2004), pp.171-203.

[5] D.M. Tanner. "Reliability of surface micromachined microelectromechanical actuators," Proc. $22^{\text {nd }}$ International Conference on Microelectronics, MIEL 2000, Nis, Serbia, 14-17 May 2000, vol. 1, pp. 97-104.

[6] M.R. Douglass. "Lifetime estimates and unique failure mechanisms of the digital micromirror device (DMD)," $36^{\text {th }}$ Annual Reliability Physics Symposium Proc., Reno, NV, 3/314/2/98, 1998 IEEE International (1998), pp. 9-16.

[7] M. Scherge, X. Li, J. A. Schaefer, "The effect of water on friction of MEMS," Tribology Lttrs., 6 (1999).

[8] N. Tambe, and B. Bhushan, "Scale dependence of micro/nanofriction and adhesion of MEMS/NEMS materials, coatings and lubricants". Nanotechnology, 15 (2004).

[9] B. Bhushan, Handbook of micro/nanotribology $2^{\text {nd }}$ ed., CRC Press, Boca Raton, FL (1999), pp. 247-272, 371-432.

[10] B. Bhushan, and C. Dandavate, "Thin-film friction and adhesion studies using atomic force microscopy," J. App. Phys., 87, 3 (2000).

[11] B.D. Terris, S.A. Rishton, H.J. Mamin, R.P. Ried, D. Rugar, "Atomic force microscope-based data storage: track servo and wear study," Appl. Phys. A, 66 (1998).

[12] D. Tabor, and R.H.S. Winterton. "The direct measurement of normal and retarded van der Waals forces," Proc. of the Royal Society of London, A, Math. and Phys.Sci. 312. (1969), pp. 435450 . 